\title{
A Recurrent Nova Super-Remnant in the Andromeda Galaxy
}

M. J. Darnley ${ }^{1}$, R. Hounsell ${ }^{2,3,4}$, T. J. O’Brien ${ }^{5}$, M. Henze ${ }^{6}$,

P. Rodríguez-Gil ${ }^{7,8}$, A. W. Shafter ${ }^{6}$, M. M. Shara ${ }^{9}$, N. M. H. Vaytet ${ }^{10,11}$,

M. F. Bode ${ }^{1,12}$, R. Ciardullo ${ }^{13,14}$, B. D. Davis ${ }^{13}$, R. Galera-Rosillo ${ }^{7,8}$,

D. J. Harman ${ }^{1}$, E. J. Harvey ${ }^{1}$, M. W. Healy ${ }^{1}$, J.-U. Ness ${ }^{15}$,

V. A. R. M. Ribeiro ${ }^{16,17}$, and S. C. Williams ${ }^{18}$

${ }^{1}$ Astrophysics Research Institute, Liverpool John Moores University, IC2 Liverpool

Science Park, Liverpool, L3 5RF, UK.

${ }^{2}$ Department of Astronomy and Astrophysics, University of California Santa Cruz, 1156

High Street, Santa Cruz, CA 95064, USA.

${ }^{3}$ Department of Astronomy, University of Illinois at Urbana-Champaign, $1002 \mathrm{~W}$. Green

Street, Urbana, IL 61801, USA.

${ }^{4}$ University of Pennsylvania, Department of Physics \& Astronomy, 209 South 33rd

Street, Philadelphia, PA 19104-6396, USA.

${ }^{5}$ Jodrell Bank Centre for Astrophysics, Alan Turing Building, University of Manchester, Manchester, M13 9PL, UK.

${ }^{6}$ Department of Astronomy, San Diego State University, San Diego, CA 92182, USA.

${ }^{7}$ Instituto de Astrofísica de Canarias, Vía Láctea, s/n, La Laguna, E-38205, Santa Cruz de Tenerife, Spain.

${ }^{8}$ Departamento de Astrofísica, Universidad de La Laguna, La Laguna, E-38206, Santa Cruz de Tenerife, Spain. 
${ }^{9}$ American Museum of Natural History, 79th Street and Central Park West, New York, NY 10024, USA.

${ }^{10}$ Centre for Star and Planet Formation, Niels Bohr Institute and Natural History Museum of Denmark, University of Copenhagen, Øster Voldgade 5-7, DK-1350 Copenhagen K, Denmark.

${ }^{11}$ Data Management and Software Centre, the European Spallation Source ERIC, Ole Maaløes Vej 3, DK-2200, Copenhagen, Denmark

${ }^{12}$ Vice Chancellor's Office, Botswana International University of Science and Technology, Private Bag 16, Palapye, Botswana.

${ }^{13}$ Department of Astronomy \& Astrophysics, The Pennsylvania State University, University Park, PA 16802

${ }^{14}$ Institute for Gravitation and the Cosmos, The Pennsylvania State University, University Park, PA 16802

${ }^{15}$ XMM-Newton Observatory SOC, European Space Astronomy Centre, Camino Bajo del Castillo s/n, Urb. Villafranca del Castillo, 28692 Villanueva de la Cañada, Madrid, Spain. ${ }^{16}$ Center for Research \& Development in Mathematics and Applications, Departamento de Física, Universidade de Aveiro, Campus Universitário de Santiago, 3810-193 Aveiro, Portugal.

${ }^{17}$ Instituto de Telecomunicações, Campus Universitário de Santiago, 3810-193 Aveiro, Portugal.

${ }^{18}$ Physics Department, Lancaster University, Lancaster, LA1 4YB, UK. 
The accretion of hydrogen onto a white dwarf star ignites a thermonuclear runaway in the accumulated envelope, leading to luminosities up to 1 million times that of the Sun and a high-velocity mass ejection that produces a remnant shell - a classical nova eruption ${ }^{1,2}$. Close to the upper mass limit of a white $\operatorname{dwarf}^{3}$ (1.4Msun), rapid accretion of hydrogen $\left(\sim 10^{-7} \mathrm{Msun} / \mathrm{yr}\right)$ from a binary star companion leads to frequent eruptions on timescales of years $^{4,5}$ to decades ${ }^{6}$. Such systems are known as recurrent novae. The ejecta of recurrent novae, initially moving at up to $10,000 \mathrm{~km} / \mathbf{s}^{(7)}$, must sweep-up the surrounding interstellar medium and evacuate cavities around the nova binary. No remnant larger than one parsec from any single classical or recurrent nova eruption is known ${ }^{8,9,10}$, but thousands of successive recurrent nova eruptions should be capable of generating shells $\sim 100-1,000$ times this size. Here we report that the most rapidly recurring nova, M31N 2008-12a, which erupts annually ${ }^{11}$, is surrounded by such a nova super-remnant with a projected size of at least 134 by 90 parsecs. Larger than almost all known remnants of supernova explosions ${ }^{12}$, this enormous shell demonstrates that M31N 2008-12a has erupted with high frequency for millions of years.

\section{Subject terms: Stars Novae}

Located within the disk of the Andromeda Galaxy (M31), the rapidly recurring nova M31N 200812a has erupted annually since at least $2008^{13}$. The eruptions of M31N 2008-12a (hereafter ' $12 \mathrm{a}$ ') exhibit the fastest optical evolution, the highest ejection velocities, the hottest X-ray source, and the most rapid recurrence cycle of any known thermonuclear nova ${ }^{11}$. Combined, these observations require the most massive white dwarf (WD) ever discovered ${ }^{14}$ (1.38Msun), accreting at the largest 
rate seen in any nova system ${ }^{15}\left(>10^{-7} \mathrm{Msun} / \mathrm{yr}\right)$. Hubble Space Telescope (HST) ultraviolet spectroscopy of the 2015 eruption uncovered no evidence for neon in the ejecta, which is consistent with - but not conclusive proof of - a carbon-oxygen $\mathrm{WD}^{16}$, one which must have grown from an initial formation mass of at most $1.1 \mathrm{Msun}^{5,17,18}$.

Pre-existing ground-based narrow-band $\mathrm{H} \alpha$ imaging shows a partially complete shell-like structure spatially coincident with the nova ${ }^{19,20}$. The full ring of this shell-like nebula surrounding $12 \mathrm{a}$ is clearly visible in newly obtained deep ground-based and HST observations (Fig. 1; see Methods) the proposed nova super-remnant. The super-remnant is elliptical and brighter to the southwest than it is to the northeast. We measure the projected semi-major axes to the inner and outer edge of the bright super-remnant shell to be 52 and $67 \mathrm{pc}$, respectively, a shell thickness of $22 \%$. If dynamic in nature, the pre-existing interstellar medium (ISM) has been swept-up and compressed by a factor of $\sim 2$. There is a sharply defined outer edge visible to the south and west. The welldefined elliptical boundary of the super-remnant implies that it has not been substantially shaped by the ISM, but that such a geometry was imparted by the nova eruptions and has largely persisted. The high spatial resolution of the HST images reveals that the super-remnant outer-shell is not smooth, as seen from the ground, but fragmented into knots and radially nested filaments, reminiscent of the handful of interacting nova shells seen around the Galactic recurrent nova T Pyxidis ${ }^{21,22}$.

Newly obtained deep spectroscopy of the super-remnant shell (see Fig. 2 and Methods) reveals strong and narrow emission lines from the hydrogen Balmer series with natural widths narrower than the instrumental resolution $(\sim 180 \mathrm{~km} / \mathrm{s}$ for $\mathrm{H} \alpha)$. The presence of the $[\mathrm{O}$ II $](3,726 / 3,729 \AA)$ and [S II] $(6,716 / 6,731 \AA)$ doublets place an upper limit on the electron density of the emitting gas of $\sim 3,000 \mathrm{~cm}^{-3(23)}$. The lack of [O III] indicates that there is no nearby source of ionising radiation and 
that the material is of sufficient age to have cooled below the ionisation temperature of $\mathrm{O}^{+}$. No [O I] lines are detected, suggesting minimal shock heating. As the [N II] $(6,548 / 6,584 \AA)$ doublet is visible, but the $[\mathrm{N} \mathrm{II}](5,755 \AA)$ line is not, we can place a $3 \sigma$ limit on the electron temperature of $<9,000 \mathrm{~K}^{(23)}$. The $[\mathrm{N} \mathrm{II}] / \mathrm{H} \alpha$ line-intensity ratio is $0.54 \pm 0.02$, the $[\mathrm{S} \mathrm{II}] / \mathrm{H} \alpha$ ratio is $0.48 \pm 0.04$, while the [S II] doublet ratio itself is $1.42 \pm 0.05$ and indicates an electron density $<100 \mathrm{~cm}^{-3(23)}$ within the bright outer-shell of the super-remnant. With a compression factor of $\sim 2$, the pre-nova ISM density must have been $<50 \mathrm{~cm}^{-3}$. This density and measurements of the super-remnant shell size (Fig. 1) indicate the shell mass is $<7 \times 10^{5} \mathrm{Msun}$ (see Methods).

A second spectrum contains emission from a bright 'knot' slightly within the outer-shell to the east of $12 \mathrm{a}$ (see Figs. $1 \mathrm{~b}$ and 2, and Methods). This is similar to, but fainter than, that of the outer-shell. However, the knot spectrum contains strong [O III] $(4,959 / 5,007 \AA)$ emission, indicating a more extreme temperature or radiation environment closer to the nova system. The lack of [O III] $(4,363 \AA)$ only allows a weak temperature constraint of $<160,000 \mathrm{~K}$, the knot $[\mathrm{N}$ II] emission indicates $\mathrm{T}<18,000 \mathrm{~K}$.

Utilising the pre-existing ground-based imaging and serendipitous spectroscopy the origin of the nebulosity could not be confirmed ${ }^{19}$. Possible sources of an elliptical nebular shell could include a supernova remnant (SNR), a 'superbubble', or photoionization phenomena, such as a fossil H II region.

The $[\mathrm{S} \mathrm{II}] / \mathrm{H} \alpha$ ratio is marginally consistent with the lower cut off $(\geq 0.5)^{24,25,26}$ required to suggest a 'forbidden line' SNR candidate, but the lack of [O III] and [O I] emission ${ }^{26}$ strongly suggests that the outer-shell is not a SNR. Moreover, there are no known radio sources close to $12 \mathrm{a}^{25,27}$ and the X-ray flux upper limit is below that of known M31 SNRs (see Methods). We conclude that the super-remnant is not a SNR. 
Superbubbles, caused by the winds of massive stars and supernovae are typically observed surrounding $\mathrm{OB}$ associations. $H S T$ observations of the region reveal no such associations within the super-remnant (see Methods) and no nearby SNRs have been identified. Known Galactic fossil $\mathrm{H}$ II regions are typically much smaller than the super-remnant. Although 12a has likely undergone eruptions for a long period, the luminosity of the eruptions is not high enough to grow a photoionized region to the size of the super-remnant (see Methods).

To demonstrate the viability of multiple recurrent nova eruptions producing a vast super-remnant, we performed a series of one-dimensional hydrodynamic simulations of the ejecta, their selfinteraction, and their interaction with the surrounding environment. Results of our simulations of up to 100,000 separate but interacting ejecta are presented in Fig. 3. The simulations (Fig. 3a-c) illustrate how repeated nova eruptions create a vast, evacuated cavity around the system, by continually sweeping up the ISM and piling it up within a shell at the edge of the growing superremnant (Fig. 3d). In Fig. 3e, the observed super-remnant radial profile is compared to the simulations (scaled to the size of the nebula), demonstrating striking similarity at scales above 10pc. These profiles are consistent with a shell rather than a ring (see Methods).

Such repeated eruptions sweep up 17Msun of ISM after 100,000 eruptions (see Methods), $\sim 3,000$ times the mass ejected by the nova over this period. Therefore, super-remnants must comprise almost exclusively swept up material and will have an ISM-like, not nova-like, composition; the super-remnant $\mathrm{He} \mathrm{I} / \mathrm{H} \alpha$ line-strength ratio of $<0.04$ is consistent with those observed in warm diffuse $\mathrm{ISM}^{28}$. The $\mathrm{He} \mathrm{I} / \mathrm{H} \alpha$ ratio of the $12 \mathrm{a}$ ejecta has been repeatedly measured and varies from $0.16-0.48^{11}$, driven by the high helium abundance of nova ejecta ${ }^{4}$. The super-remnant contains three distinct regions, as marked in Fig. 3b (also see Methods): the inner cavity, where recent ejecta effectively undergo free, high-velocity expansion while cooling 
adiabatically; the ejecta pile-up, where the ejecta from successive eruptions eventually collide as preceding eruptions are slowed by interaction with the ISM, with high-velocity inter-ejecta shocks driving significant heating and deceleration of this gas; and the super-remnant shell, which consists almost entirely of swept-up ISM that is slowly driven outward by the multiple-ejecta pileup culminating at its inner edge. The radii of the outer and inner edges of the super-remnant increase with a power-law-like time dependence (Fig. 3d), maintaining a shell thickness of $\sim 22 \%$, consistent with the observations. Once established, the peak shell over-density remains at four times the ISM density (Fig. 3a-c).

The computational intensity limited simulations to 100,000 eruptions; continued growth of the super-remnant has been explored by extrapolation to later times (see Methods). Our models show that the 12a super-remnant has been built up by annual nova eruptions sweeping up the surrounding ISM over $6 \times 10^{6}$ years (Fig. 3d). Over that time, the outer-shell of the super-remnant has cooled sufficiently below $10^{4} \mathrm{~K}$ to explain the observed spectrum (Fig. $3 \mathrm{f}$ ). Throughout the evolution, the high temperature of the pile-up region is however maintained by the continual arrival of high-velocity ejecta, a possible explanation of the inner knot spectrum. The predicted X-ray luminosity of the super-remnant is orders of magnitude below current detection capabilities (see Methods). The simulations show that the total mass swept up by the eruptions is $\sim 3 \times 10^{4}$ Msun, consistent with the upper limit derived from the observations. The size and mass of this super-remnant demonstrate that $12 \mathrm{a}$ has not just been erupting frequently for a decade as observed, but for millions of years.

The 12a WD has an accretion rate of $\sim 1.6 \times 10^{-7} \mathrm{Msun} / \mathrm{yr}^{(14)}$ and a current accretion efficiency (the proportion of accreted material retained by the WD post-eruption) $>60 \%{ }^{14,15}$. Assuming a WD formation mass of $\sim 1$ Msun, an average efficiency of just $40 \%$ over the age of the remnant is 
required to grow the WD to the maximum mass permissible before collapse ensues ${ }^{3}(1.4 \mathrm{Msun}$; the Chandrasekhar limit). This is consistent with predictions of increasing accretion efficiency as the WD mass grows 5 .

The discovery of additional super-remnants around other accreting WDs will provide striking signposts to systems undergoing regular eruptions over long periods of time. Our simulations show that this super-remnant, the first discovered extragalactic nova shell, is not static and will continue

to grow at least as long as nova eruptions continue in the system. Any nova super-remnants around accreting carbon-oxygen WDs will ultimately be destroyed by the explosion of their parent system as a Type Ia supernova. M31N 2008-12a is predicted to surpass the Chandrasekhar limit in less than 40,000 years ${ }^{15}$. At such time, the underlying composition of the $\mathrm{WD}^{16}$ will be revealed incontrovertibly when either a Type Ia supernova ${ }^{29}$, or an accretion induced collapse of the WD to a neutron $\operatorname{star}^{30}$, is observed.

\section{References}

1. Starrfield, S., Truran, J. W., Sparks, W. M., Kutter, G. S. CNO Abundances and Hydrodynamic Models of the Nova Outburst. Astrophys J. 176, 169-176 (1972).

2. Prialnik, D., Shara, M. M., Shaviv, G. The evolution of a slow nova model with a $\mathrm{Z}=0.03$ envelope from pre-explosion to extinction. Astron. Astrophys. 62, 339-348 (1978).

3. Chandrasekhar, S. The Maximum Mass of Ideal White Dwarfs. Astrophys J. 74, 81-82 (1931).

4. Kato, M., Saio, H., Hachisu, I, Nomoto, K. Shortest Recurrence Periods of Novae. Astrophys J. 793, 136 (2014). 
5. Hillman, Y., Prialnik, D., Kovetz, A., Shara, M. M. Growing White Dwarfs to the Chandrasekhar Limit: The Parameter Space of the Single Degenerate SN Ia Channel. Astrophys J. 819, 168 (2016).

6. Schaefer, B. Comprehensive Photometric Histories of all Known Galactic Recurrent Novae. Astrophys J. Suppl. Ser. 187, 275-373 (2010).

7. Munari, U., Zwitter, T., Tomov, T., Bonifacio, P., Molaro, P., et al. The 1999 outburst of the eclipsing and recurrent nova U Scorpii. Astron. Astrophys. 347, L39-L42 (1999). 8. Bode, M. F., O’Brien, T. J., Simpson, M. Echoes of an explosive past: Solving the mystery of the first superluminal source. Astrophys. J. 600, L63-L66 (2004).

9. Shara, M. M., Martin, C. D., Seibert, M., Rich, R. M., Salim, S., An ancient nova shell around the dwarf nova Z Camelopardalis. Nature. 446, 159-162 (2007).

10. Shara, M. M., Mizusawa, T., Wehinger, P., Zurek, D., Martin, C. D., et al. AT Cnc: A Second Dwarf Nova with a Classical Nova Shell. Astrophys. J. 758, 121-125 (2012).

11. Darnley, M. J., Henze, M., Bode, M. F., Hachisu, I., Hernanz, M., et al. M31N 2008-12a — The Remarkable Recurrent Nova in M31: Panchromatic Observations of the 2015 Eruption. Astrophys J. 833, 149 (2016).

12. Stil, J. M., Irwin, J. A. GSH 138-01-94: An old Supernova Remnant in the Far Outer Galaxy. Astrophys J. 563, 816-827 (2001).

13. Henze. M., Darnley, M. J., Williams, S. C., Kato, M., Izumi, H., et al. Breaking the habit — the peculiar 2016 eruption of the unique recurrent nova M31N 2008-12a. Astrophys. J. 857, 68-96 (2018).

14. Kato, M., Saio, H., Hachisu, I., Multi-Wavelength Light Curve Model of the One Year Recurrence Period Nova M31N 2008-12a. Astrophys J. 805, 52 (2015). 
15. Darnley, M. J., Hounsell, R., Godon, P., Perley, D. A., Henze, M., et al. Inflows, Outflows, and a Giant Donor in the Remarkable Recurrent Nova M31N 2008-12a?Hubble Space Telescope Photometry of the 2015 Eruption. Astrophys J. 849, 96 (2017). 16. Darnley, M. J., Hounsell, R., Godon, P., Perley, D. A., Henze, M., et al. No Neon, but Jets in the Remarkable Recurrent Nova M31N 2008-12a?-Hubble Space Telescope Spectroscopy of the 2015 Eruption. Astrophys J. 847, 35 (2017).

17. Toonen, S., Voss, R., Knigge, C. The influence of mass-transfer variability on the growth of white dwarfs, and the implications for Type Ia supernova rates. Mon. Not. R. Astron. Soc. 441, 354-363 (2014).

18. Ritossa, C., García-Berro, E., Iben, I. On the Evolution of Stars That Form Electron-degenerate Cores Processed by Carbon Burning. II. Isotope Abundances and Thermal Pulses in a 10 Msun Model with an ONe Core and Applications to Long-Period Variables, Classical Novae, and Accretion-induced Collapse. Astrophys J. 460, 489-505 (1996).

19. Darnley, M. J., Henze, M., Steele, I. A., Bode, M. F., Ribeiro, V. A. R. M., et al. A remarkable recurrent nova in M31: Discovery and optical/UV observations of the predicted 2014 eruption. Astron. Astrophys. 580, A45 (2015).

20. Walterbos, R. A. M., Braun, R. The interstellar medium of M31: III. Narrow-band imagery in Ha and [SII]. Astron. Astrophys. Suppl. Ser. 92, 625-682 (1992).

21. Shara, M. M., Zurek, D., Williams, R. E., Prialnik, D. Gilmozzi, R., Moffat, A. F. J. HST Imagery of the Non-expanding, Clumped "Shell” of the Recurrent Nova T Pyxidis. Astron. J. 114, 258-275 (1997). 
22. Toraskar, J., Mac Low, M.-M., Shara, M. M., Zurek, D. R. Dynamical Fragmentation of the T Pyxidis Nova Shell During Recurrent Eruptions. Astrophys J. 768, 48 (2013).

23. Osterbrock, D. E., Ferland, G. J. Astrophysics of gaseous nebulae and active galactic nuclei, 2nd edition. (Sausalito, CA: University Science Books, 2006).

24. Kniazev, A. Y., Pustilnik, S. A., Zucker, D. B. Spectroscopy of two PN candidates in IC10. Mon. Not. R. Astron. Soc. 384, 1045-1052 (2008).

25. Sasaki, M., Pietsch, W., Haberl, F., Hatzidimitriou, D., Stiele, H. Supernova remnants and candidates detected in the XMM-Newton M31 large survey. Astron. Astrophys. 544, A144 (2012).

26. Long, K. S., Blair, W. P., Kirshner, R. P., Winkler, P. F. An Atlas of Confirmed and Candidate Supernova Remnants in M33. Astrophys J. Suppl. Ser. 72, 61-73 (1990).

27. Braun, R., Walterbos, R. A. M. An atlas of supernova remnant candidates in Messier 31. Astron. Astrophys. Suppl. Ser. 98, 327-364 (1993).

28. Reynolds, R. J., Tufte, S. L. A search for the He I $\lambda 5876$ recombination line from the diffuse interstellar medium. Astrophys J. 439, L17-L20 (1995).

29. Whelan, J., Iben, I. Binaries and Supernovae of Type I. Astrophys J. 186, 1007-1014 (1973).

30. Gutiérrez, J., García-Berro, E., Iben, I. Isern, J., Labay, J., Canal, R. The Final Evolution of ONeMg Electron-Degenerate Cores. Astrophys J. 459, 701-705 (1996). 


\section{Acknowledgments:}

The Liverpool Telescope is operated on the island of La Palma by Liverpool John Moores University in the Spanish Observatorio del Roque de los Muchachos of the Instituto de Astrofísica de Canarias with financial support from STFC.

Based on observations made with the NASA/ESA Hubble Space Telescope, obtained from the Data Archive at the Space Telescope Science Institute (STScI), which is operated by the Association of Universities for Research in Astronomy (AURA) under NASA contract NAS 5-26555. These observations are associated with programmes GO:14125 and GO:14651 for which financial support for RH, MH, MMS, and AWS was provided by NASA through grants from STScI.

Based on observations made with the Gran Telescopio Canarias, installed in the Spanish Observatorio del Roque de los Muchachos of the Instituto de Astrofísica de Canarias, in the island of La Palma.

The Hobby-Eberly Telescope is a joint project of the University of Texas at Austin, the Pennsylvania State University, Stanford University, Ludwig-Maximilians-Universität München, and Georg-August-Universität Göttingen. HET is named in honour of its principal benefactors, William P. Hobby and Robert E. Eberly.

The authors thank Z. Levay for creating a colour composite image of the nova superremnant, I. A. Steele for assistance with the LT spectra, K. L. Page for assistance with XSPEC, K. A. Misselt and D. Baer for assistance with the Steward 2.3m observations, also M. Link and C. Proffitt, and W. Eck and K. Long, the programme coordinators and contact scientists for HST GO:14124 and GO:14651, respectively. 
MJD and MWH acknowledge financial support and a PhD studentship, respectively, from STFC.

NMHV acknowledges support from the European Commission through the Horizon 2020 Marie Sklodowska-Curie Actions Individual Fellowship 2014 programme (Grant Agreement no. 659706).

VARMR acknowledges financial support from Fundação para a Ciência e a Tecnologia (FCT) in the form of an exploratory project of reference IF/00498/2015, from the Center for Research \& Development in Mathematics and Applications strategic project UID/MAT/04106/2013, and from Enabling Green E-science for the Square Kilometer Array Research Infrastructure (ENGAGE SKA), POCI-01-0145-FEDER- 022217, funded by Programa Operacional Competitividade e Internacionalização (COMPETE 2020) and FCT, Portugal.

\section{Author contributions:}

All authors contributed to the discussion, proposing and planning of observations, data interpretation, and writing of this manuscript. MJD and SCW led the Liverpool Telescope observations. MJD and RH wrote the proposals and led the Hubble Space Telescope observations and resulting analysis. PR-G and MJD proposed and led the Gran Telescopio Canarias observations, RG-R assisted with their analysis. RC and BDD obtained the Hobby Eberly Telescope spectrum. AWS acquired the Steward 2.3m Bok Telescope data. MH analysed the archival X-ray data. MJD, MWH, and SCW undertook the photoionization analysis. MJD, TJO'B, and NMHV led the hydrodynamic simulations. MJD and NMHV produced the synthetic X-ray spectra. 


\section{Competing Financial Interests}

The authors declare no competing financial interests.

\section{Correspondence Author}

Correspondence and requests for materials should be addressed should be addressed to MJD (M.J.Darnley@ljmu.ac.uk).

\section{Main Figure Legends}

Figure 1. M31N 2008-12a and its surrounding super remnant. (a), Liverpool

Telescope narrow-band $\mathrm{H} \alpha+[\mathrm{N}$ II $]$ continuum-subtracted (see Methods) image of the region surrounding M31N 2008-12a. The majority of stellar sources have been removed, but the eight dark-blue sources indicate field stars only detected in continuum light. The presence of the closed nebula is seen within the white dashed ellipse, as is its asymmetry and varying luminosity around the outer 'shell'. The position of M31N 2008-12a is marked and the offset from the geometric centre is indicated by the black line. (b), Hubble Space Telescope $\mathrm{H \alpha}+[\mathrm{N} \mathrm{II}]$ continuum-subtracted (see Methods) image of the same region; all stellar sources have been removed via the subtraction process. The high spatial resolution of this image reveals that the nebulosity is not smooth as imaged from the ground, but fragmented and filamentary in nature, reminiscent of the ejecta of the Galactic recurrent nova T Pyxidis ${ }^{22}$. The red squares mark the location of the two regions discussed in the text; the large square shows the bright western shell, and the small square the eastern 'knot'. (c), zoomed in Hubble Space Telescope $\mathrm{H} \alpha+[\mathrm{N}$ II] image showing the 
region within the large red box in the centre panel. To the top of this panel three long 'nested' filaments are discernible, separated by only 5 and $12 \mathrm{pc}$, respectively.

Figure 2. Spectroscopy of the super remnant shell. The Gran Telescopio Canarias spectrum (top; black) of the bright western part of the nova super-remnant shell (position as indicated in Fig. 1). This spectrum shows negligible continuum emission punctuated

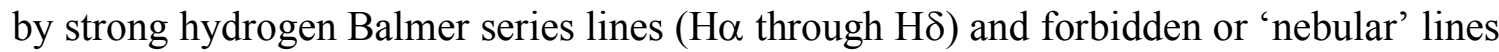
of [N II], [O II], and [S II]. With a spectral resolution of 5.3 $\AA$, the $\mathrm{H} \alpha+[\mathrm{N}$ II] and [S II] lines are easily resolved, but the [O II] doublet is blended. No other lines can be reliably confirmed. The lower spectrum (grey) is that of the inner eastern knot (see Fig. 1). This spectrum has been shifted down in flux by $2 \times 10^{-17} \mathrm{erg} \mathrm{s}^{-1} \mathrm{~cm}^{-2} \AA^{-1}$ for clarity. All lines

visible in the outer-shell spectrum are present, but they have been joined by strong [O III] $(4,959 / 5,007 \AA)$ lines. Gaps in the spectra indicate areas where significant skyline subtraction residuals remained. Both spectra have been flux calibrated relative to the Hobby Eberly Telescope spectrum of the bright western part of the super-remnant shell (see Methods).

Figure 3. Results of hydrodynamic simulations of the interacting ejecta of multiple recurrent nova eruptions. (a-c), the radial density profile around M31N 2008-12a. The solid lines illustrate the simulated density profile from 2-100,000 eruptions (see respective keys). The lower and upper dotted lines show the ISM and outer-shell peak densities, respectively. (d), the upper solid line illustrates the growth of the outer edge of the super-remnant shell over 100,000 eruptions, the lower solid line shows the inner edge growth. The diagonal dotted lines are extrapolations of the radial growth curves to further eruptions. The upper/lower grey lines indicate the growth of the outer edge for 
lower/higher ISM densities, respectively. The horizontal dotted line is the maximum projected radius of the M31N 2008-12a super-remnant (67pc; the 45pc semi-minor axis is also shown). (e), the radial $\mathrm{H} \alpha+[\mathrm{N}$ II] flux from the LT (grey) and $H S T$ (red) imaging compared to the simulated super-remnant hydrogen column (black). The simulation has been rescaled from 100,000 eruptions to the observed size of the remnant. (f), the super-remnant temperature evolution. The solid black line indicates simulations of 100,000 eruptions, the red/green lines show the effects of a lower/higher ISM density, respectively. An extrapolation to further eruptions is shown by the diagonal black dotted line, with the current predicted shell temperature of $\sim 1,200 \mathrm{~K}$ indicated to the lower-right. The solid blue line indicates the evolution of the mean electron temperature within the ejecta pile-up region. The horizontal lines indicate the shell electron temperature upper limit as required by the spectroscopy; and the ionisation temperatures of $\mathrm{O}^{+}$and $\mathrm{N}^{+}$, as required to observe [O III] or [N III] lines in the spectra.

\section{Methods}

\section{M31N 2008-12a}

The recurrent nova M31N 2008-12a (hereafter ' $12 \mathrm{a}$ ') is located in north-eastern part of the outer disk of M31 with equatorial coordinates $0^{\mathrm{h}} 45^{\mathrm{m}} 28.89^{\mathrm{s}}+41^{\circ} 54^{\prime} 10.2^{\prime \prime}(\mathrm{J} 2000)^{31}$. Eruptions have been detected in each year from 2008 to 2017 and recovered from archival X-ray observations taken in 1992, 1993, and 2001 ${ }^{32}$. The 2013-2017 eruptions have been studied extensively in the X-ray, far- and near-ultraviolet, and optical. The recurrence period of the system is $347 \pm 10$ days ${ }^{11}$, although an alias of $174 \pm 10$ days ${ }^{33}$ cannot yet be entirely excluded. The mass donor has been identified as a 'red clump' 
star $^{15}$, but based on Galactic recurrent nova systems ${ }^{6}$ is most likely to be a low-luminosity red giant, with accretion driven either by Roche lobe overflow or by the red giant wind. Spectroscopy of the 2012-2017 eruptions has shown strong evidence for the deceleration of the 12a ejecta over the first 5 days post-eruption as they interact with and shock circumbinary material, which must be replenished between each eruption ${ }^{11,19}$. The most likely source of this material is from a donor wind, although an accretion disk wind has also been proposed ${ }^{15}$.

Short recurrence times are driven by the combination of a high-mass WD and a high mass accretion rate ${ }^{4}$. Among Galactic systems, U Scorpii exhibits the shortest recurrence period of $\sim 10$ years $^{6}$, although recently a number of other short-period $(<10$ years, but $>1$ year) systems have been discovered in $\mathrm{M} 31^{34}$.

\section{Ground-based imaging observations}

Nebulosity in the region around 12a had first been identified as a 'ring' like structure as part of a narrow-band survey of M31 undertaken in $1987^{20}, 21$ years before the first optical eruption was discovered. Following the 2015 eruption of 12a, an inspection of $\mathrm{H \alpha}$ data collected using the Steward 2.3m Bok Telescope in 2005 and 2006 (see Extended Data Fig. 1a) marked the 'rediscovery' of the nebulosity but its first association with the recurrent nova ${ }^{19}$. A further series of $20 \times 180$ s narrow-band $\mathrm{H} \alpha$ images of the nebula were taken using the IO:O CCD camera on the $2.0 \mathrm{~m}$ fully robotic Liverpool Telescope (LT ${ }^{35}$ ) on 2014 July $30^{19}$. The narrow-band H $\alpha$ filter on the LT has a bandpass full-width at half-maximum (FWHM) of $100 \AA$ and therefore contains $\mathrm{H} \alpha+[\mathrm{N}$ II] emission.

Subsequently, those data were supplemented by an additional series of $20 \times 180$ s $\mathrm{H} \alpha+[\mathrm{N}$ II] images taken between the 2014 and 2015 eruptions of $12 \mathrm{a}$ and by $66 \times 300 \mathrm{~s}$ 
$\mathrm{H} \alpha+\left[\mathrm{N}\right.$ II] images obtained during the 2014 eruption $^{19}$. When combined, these produced a new deep $27 \mathrm{ks} \mathrm{H} \alpha+[\mathrm{N}$ II] image of $12 \mathrm{a}$ and the surrounding region. A deep (55.6ks) broad-band Sloan $r^{\prime}$-band image of the region was also produced by combining all available LT observations of the 2014 and 2015 eruption campaigns ${ }^{11,19}$. These data were processed, co-aligned, and stacked using standard tools within the IRAF $^{36}$ environment. DAOPHOT ${ }^{37}$ was then utilised to perform photometry on all sources common to the $\mathrm{H \alpha}+[\mathrm{N}$ II $]$ and $r^{\prime}$ images, which were then removed, before photometrically aligning the data, and subtracting. The resultant continuum-subtracted $\mathrm{H} \alpha+[\mathrm{N} \mathrm{II}]$ image is shown in Fig. 1a.

The radial profile of the super-remnant was produced by performing annular photometry of the region, centred on the apparent geometric centre of the remnant $\left(0^{\mathrm{h}} 45^{\mathrm{m}} 28.6^{\mathrm{s}}\right.$ $\left.+41^{\circ} 54^{\prime} 13^{\prime \prime}\right)$, using elliptical annuli with an axial ratio of $3: 2$ and position angle of $40^{\circ}$. The radial profile of the LT data out to $\sim 550 \mathrm{pc}$ from 12a is shown in Fig. 3e. This figure clearly indicates the peak in $\mathrm{H} \alpha+[\mathrm{N}$ II $]$ flux at $\sim 67 \mathrm{pc}$, but also the flux from within the super-remnant, compared with the surrounding region in M31 (effectively zero). The $\mathrm{H} \alpha+[\mathrm{N} \mathrm{II}]$ emission from the inner region is approximately $1 / 3$ that of the outer-shell. This implies that the observed phenomenon is a three-dimensional limb-brightened shell and not a two-dimensional ring approximately aligned to the plane of the sky.

\section{Hubble Space Telescope observations}

Ten orbits of Cycle $24 H S T$ time were used to obtain $\mathrm{H \alpha}+[\mathrm{N}$ II $]$ imaging of the nebulosity around 12a (GO:14651). These observations were conducted on 2016 December 7, 8, 9, 10,11 , and 17 . By chance, the 2016 eruption of $12 \mathrm{a}$ was captured in those observations on December $17^{13}$. 
The orbits were split into five pairs, with each pair collecting narrow-band imaging through the $\mathrm{F} 675 \mathrm{~N}(\mathrm{H \alpha}+[\mathrm{N} \mathrm{II}])$ filter for one orbit, and through the $\mathrm{F} 645 \mathrm{~N}$ filter (for continuum subtraction) for the second orbit; both utilising the UVIS mode of the Wide Field Camera 3 instrument. The total exposure time for each F675N and F645N orbit was 2,694s and 2,805s, respectively. The light-grey shaded region in Fig. 2 indicates the FWHM of the F645N filter, confirming that it is not affected by any line emission from the super-remnant.

For each filter, a three-point dither was applied to enable the removal of detector defects and cosmic ray rejection. The dither pattern of each visit was further offset to allow for enhanced spatial resolution. A post-flash signal of 12 electrons was included to minimise losses due to non-ideal charge transfer efficiency. These data were reduced using the STScI calwfc pipeline ${ }^{38}$, and Drizzlpac was used to align and combine the data to create the final images (Fig. 1b \& 1c) with spatial resolution of $0.0333^{\prime \prime} /$ pixel $(\sim 0.1 \mathrm{pc}$ at the distance of M31). Continuum subtraction was achieved by flux aligning the images using resolved stellar sources in both the F645N and F675N images. As 12a itself was detected in all five observations (and in eruption in one of those) and is an $\mathrm{H} \alpha$ emitter during eruption and quiescence, this method did not subtract 12a itself from these images. The super-remnant radial profile based on the $H S T$ observations was produced in a similar manner for that from the LT, the $H S T$ data have been truncated at $2 \mathrm{pc}$ to remove the effect of the eruption of 12a.

HST Wide Field Camera 3 images of the region around 12a were taken through broadband optical and ultraviolet filters during cycle $23(\mathrm{GO}: 14125)^{15}$. These images were processed and combined in a similar fashion to the narrow-band $\mathrm{H} \alpha+[\mathrm{N}$ II] images. A 
sample of these images is shown in Extended Data Fig. 1b-d. Consistent with the groundbased spectra, there is no evidence for any continuum emission from any part of the super-remnant in these images, nor any strong UV sources (besides 12a).

\section{Ground-based spectroscopic observations}

Five 30-minute spectra spanning the wavelength range from 3,670 to $7,870 \AA$ were taken with the OSIRIS instrument on the Gran Telescopio Canarias on 2017 January 16. We used a slit width of $0.6^{\prime \prime}$ orientated east-west and the 'R1000B' grism, achieving a spectral resolution of $5.3 \AA(\sim 250 \mathrm{~km} / \mathrm{s}$ for $\mathrm{H} \alpha)$. After image reduction, cosmic ray, and two-dimensional sky background removal using IRAF, the five spectra were co-added, and the one-dimensional spectra of the super-remnant shell and eastern 'knot' were optimally extracted using PAMELA (part of the Starlink software package ${ }^{39}$ ). On 2018 January 12, the super-remnant was observed for $2 \times 757$ s through $2.2^{\prime \prime}$ seeing with the blue feed of the new integral-field unit (IFU) low-resolution spectrograph $(\text { LRS2-B) })^{40}$ on the Hobby-Eberly Telescope (HET). This double-armed instrument surveys a $12^{\prime \prime} \times 6^{\prime \prime}$ area on the sky using an array of $22 \times 13$ lenslet-coupled fibres, and produces an image scale of 0.59 "/fibre. The "Blue Arm" of LRS2-B covers the wavelength range $3700-4700 \AA$ at $\mathrm{R} \sim 1,910$ resolution, while the "Orange Arm" simultaneously records the region $4,600-7,000 \AA$ at $\mathrm{R} \sim 1140$. We note that because LRS2-B employs lenslets, there are no dead spots between the fibres, hence many of the problems associated with faint-object spectrophotometry - such as atmospheric dispersion, slit losses, and lack of data acquisition due to imprecise astrometry - are mitigated. The HET data were reduced using the procedures described in (ref. 41), flux calibration was achieved via comparison to the tertiary spectrophotometric standard star 
HD 289002 ${ }^{(42)}$. By comparing the emission line fluxes between the outer-shell spectra from HET (flux calibrated) and GTC, an approximate flux calibration was applied to both the GTC spectra.

In Fig. 2 the flux calibrated GTC spectra of the super-remnant outer-shell (black) and the inner eastern knot (grey) are presented. In Extended Data Fig. 2 we show the HET spectrum of the outer-shell. In addition to hydrogen Balmer series lines, the GTC spectrum of the outer-shell included emission lines from the resolved [N II] $(6,548 / 6,584 \AA)$ and [S II] $(6,716 / 6,731 \AA)$ doublets, the unresolved [O II] $(3,726 / 3,729 \AA)$ doublet can be seen, all on top of a negligible continuum flux. The higher spectral resolution, but lower S/N, HET spectrum resolved the [O II] doublet (see Extended Data Fig. 2 inset). The [O II] doublet ratio ([O II] 3,729/[O II ] 3,726) is $1.5 \pm 0.3$, consistent with the outer-shell density limit of $<100 \mathrm{~cm}^{-3(23)}$, as derived from the [S II] doublet. There is little evidence for any other species, including the forbidden lines of O I or O III - if seen, both are indicative of shock heating. There are no He lines observed.

While the inner 'knot' spectrum is broadly similar to that of the super-remnant shell it is significantly fainter (Fig. 2). The [O II] emission in the knot has been joined by strong [O III] $(4,959 / 5,007 \AA)$ emission. The knot [O III] $(5,007 \AA)$ line intensity surpasses that of $\mathrm{H} \beta$, and the $[\mathrm{N} \mathrm{II}](6,584 \AA)$ line is as strong as $\mathrm{H} \alpha$. The [O III] $(4,363 \AA)$ line and again the $[\mathrm{N}$ II] $(5,755 \AA)$ line are not observed. The $[\mathrm{N} \mathrm{II}] / \mathrm{H} \alpha$ ratio in the knot is $1.23 \pm 0.08$, and $[\mathrm{S} \mathrm{II}] / \mathrm{H \alpha}=1.54 \pm 0.09$, the $[\mathrm{S} \mathrm{II}]$ doublet ratio ([S II] 6,717/[S II] 6,731) is $1.41 \pm 0.07$, and the $[\mathrm{O}$ III $](5,007 \AA) / \mathrm{H} \beta$ ratio is $1.8 \pm 0.5$. The line ratios in the knot spectrum indicate a similar density upper limit to the super-remnant shell. However, these ratios also point toward the inner knot containing much more strongly ionised gas. 
Given its location within the super-remnant, it is plausible that the knot spectrum contains contributions from both the outer-shell (giving rise to the Balmer and [O II] emission, and a contribution to the $[\mathrm{N} \mathrm{II}]$ and $[\mathrm{S} \mathrm{II}]$ ) and the hotter transition region between outer-shell and the ejecta pile-up region (leading to the [O III] and enhanced [N II] and [S II] emission).

\section{The shell mass, luminosity, and the motion of M31N 2008-12a}

The upper limit on the shell mass of the super-remnant was estimated from the imaging and spectroscopy. Based on studies of Galactic nova shells ${ }^{43}$ we assumed a bi-axial geometry (either prolate or oblate). We used the projected semi-major and semi-minor axes of 67 and $45 \mathrm{pc}$, respectively, a shell thickness of $22 \%$, and the [S II] electron density upper limit of $100 \mathrm{~cm}^{-3}$. We note that the measured shell thickness ratio is invariant to projection effects. We derive shell mass upper limits of $7 \times 10^{5} \mathrm{M}_{\text {sun }}$ and $10^{6} \mathrm{M}_{\text {sun }}$ for prolate and oblate geometries, respectively. We also note that the [S II] doublet is not a sensitive probe of densities below $100 \mathrm{~cm}^{-3(23)}$.

Using the $H S T$ continuum-subtracted $\mathrm{H} \alpha+[\mathrm{N}$ II] image (Fig. 1b) we computed the integrated $\mathrm{H \alpha}+[\mathrm{N} \mathrm{II}]$ flux from the super-remnant to be $7 \times 10^{-17} \mathrm{~W} \mathrm{~m}^{-2}$. When accounting for the distance to $\mathrm{M} 31$ of $770 \pm 19 \mathrm{kpc}^{44}$, we find that the total $\mathrm{H \alpha}+[\mathrm{N}$ II] luminosity alone from the super-remnant is $1,300 \pm 200$ Lsun (bolometric). In Fig. 1 we show that 12a is offset from the geometric centre of the super-remnant by $13 \mathrm{pc}$. The geometric centre was determined by the best-fitting ellipse to the optical imaging (see Fig. 1a). To attain such a displacement over $6 \times 10^{6}$ years a transverse velocity of $2.1 \mathrm{~km} / \mathrm{s}$ is required. All the spectra of $12 \mathrm{a}$ in eruption show no evidence for a significant $(\geq 100 \mathrm{~km} / \mathrm{s})$ radial component to the system velocity ${ }^{11,13,16,19,31}$. 


\section{Hydrodynamic modelling}

The hydrodynamic simulations were performed with the Morpheus program, an MPIOpenMP Eulerian second-order Godunov simulation code with options of Cartesian, spherical and cylindrical coordinates which includes radiative cooling and gravity. Morpheus combines well-established 1D (Asphere $\left.{ }^{45}\right), 2 \mathrm{D}\left(\right.$ Novarot $\left.^{46}\right)$ and 3D $\left(\mathrm{CubeMPI}^{47}\right)$ codes written by the Manchester-LJMU astrophysics groups into a single framework. For the purposes of these simulations we assumed one-dimensional spherical symmetry.

Based on observations of $12 \mathrm{a}$ and theoretical modelling of the eruptions ${ }^{11,14,15,19}$, we assumed the following model for the system: The mass donor is a red giant with a wind mass loss rate (after any accretion on to the WD) of $2.6 \times 10^{-8} \mathrm{Msun} / \mathrm{year}$, the terminal velocity of the red giant wind is $20 \mathrm{~km} / \mathrm{s}$ (cf. RS Ophiuchi ${ }^{48}$ ), and this wind blows continuously, except during the eruption period. The mass loss from the WD, via nova eruptions, is modelled as a wind with a constant mass-loss rate and velocity which has a simple top-hat function 'on' for 7 consecutive days in every 350 days (the nova recurrence period). The total mass ejected by each nova eruption is $5 \times 10^{-8} \mathrm{Msun}$, the ejecta have a terminal velocity of $3,000 \mathrm{~km} / \mathrm{s}$. By injecting mass with terminal velocity, we can neglect gravity. As the spatial resolution of the larger simulations is smaller than the expected orbital separation, both the donor star and the WD are assumed to be spatially coincident at the origin and there is assumed to be no interaction between the ejecta and the donor or the accretion disk. Mass injection via a wind or nova ejecta is effected by means of a boundary condition at the inner boundary of the simulation grid. The energy of the injected mass is dominated by kinetic energy. The grid is uniformly 
spaced and the maximum domain size is predetermined to contain the outer edge of the super-remnant. All simulations have sufficient spatial resolution to resolve and follow each separate eruption until they merge in the pile-up region. For computational efficiency, the domain is actively resized as the super-remnant grows by the addition of new cells.

Simulations were conducted as follows: An initial run, following 20 eruptions, with a spatial resolution of $0.02 \mathrm{AU}$ per cell and maximum domain size $10^{17} \mathrm{~cm}(6,667 \mathrm{AU})$ was conducted to 'bench-mark' the lower-resolution simulations. This was followed by a simulation of 100 eruptions with $0.2 \mathrm{AU} / \mathrm{cell}$ and maximum domain $3 \times 10^{17} \mathrm{~cm}, 1,000$ eruptions at $0.4 \mathrm{AU} / \mathrm{cell}$ and maximum domain $1.2 \times 10^{18} \mathrm{~cm}, 10,000$ eruptions with a resolution of $1 \mathrm{AU} / \mathrm{cell}$ and maximum domain $4.4 \times 10^{18} \mathrm{~cm}(1.43 \mathrm{pc})$, and finally, 100,000 eruptions with $4 \mathrm{AU} / \mathrm{cell}$ resolution and domain size $1.8 \times 10^{19} \mathrm{~cm}(5.8 \mathrm{pc})$. All of these simulations prepopulated the domain with a low pressure, cold (90K), uniform ISM density of 1 hydrogen atom per $\mathrm{cm}^{3}$, and did not invoke radiative cooling. Prepopulating the domain with a red giant wind did not make any significant differences after $\sim 10$ eruptions. Extended Data Fig. 3 illustrates the consistency between the models at all resolutions, particularly within the outer dense shell of the super-remnant. Simulations of 100 eruptions were also conducted, as above, to explore the effect of different ISM densities on the super-remnant. This effect is shown in Fig. 3d. As any energy lost due to radiative cooling can affect the dynamics of a system, a simulation of 1,000 eruptions, again as above, was also conducted while utilising the radiative cooling module of Morpheus with a suitable cooling curve ${ }^{45}$. The results of this simulation are presented in Extended Data Fig. 4 and are compared to the uncooled version. Again, 
although the details of the freely expanding nova ejecta are slightly altered, the grossstructure of the super-remnant shell is consistent. We also note that radiative cooling is inefficient above $10^{6.5} \mathrm{~K}$ and for low densities, which is the case for all the material in the remnant shell and the ejecta pile-up regions. Therefore, for computational ease, we moved on to simulate greater numbers of ejecta while ignoring radiative cooling effects. Fig. 3 f shows that eventually the super-remnant shell will cool sufficiently that radiative cooling may be important, however, the efficiency of such cooling also depends on the square of the density, as the shell cools further, below $10^{4} \mathrm{~K}$ cooling becomes inefficient once more, so we do not expect cooling to greatly affect the results at later times. As the addition of the Morpheus radiative cooling module had negligible effect on the results of the simulations, we can conclude that radiative losses must be minimal and do not affect the dynamics of the system.

The remnant mass (Extended Data Fig. 5a) was computed by integrating over the superremnant shell, defined as the outer region whose density is above that of the ISM (see Fig. 3a-c). Extrapolation of the simulations to greater time-scales was performed by fitting the super-remnant growth curves, as shown in Fig. 3d and Extended Data Fig. 5a, with power-laws.

The predicted radial profile of the simulated super-remnant (Fig. 3e) was produced by integrating the computed density profile over a sphere and then generating a collapsed two-dimensional image. The resultant image was smoothed to match the point-spread function (PSF) of HST and photometry was performed on this image in-line with the radial profiles of the LT and $H S T$ data. Simulation data have been truncated at $10 \mathrm{pc}$ to remove the effect of subsequently smoothed eruptions of $12 \mathrm{a}$. We note the agreement 
between this simulated profile of a shell-like nebula and the radial profiles from the LT and HST data.

These simulations using perfectly periodic eruptions of $12 \mathrm{a}$ lead to the production of an extremely smooth super-remnant outer-shell and ejecta-pile up region (see Fig. $3 \mathrm{c}$ and Extended Data Fig. 6 in particular), which is potentially at odds with the HST observations (Fig. 1c). However, over the past decade, the inter-eruption time of 12a has varied by \pm 52 days ${ }^{(13)}$. As such, we performed an additional simulation of 1,000 eruptions with a Gaussian distribution of onset times, with mean 350 days and sigma 52 days. The gross results of this simulation are entirely consistent with those of the periodic models. However, non-periodic eruptions alone produce potentially observable substructure within the outer-shell of the super-remnant.

\section{Photoionization analysis}

A 'fossil H II' region refers to a region of the ISM which is ionized by photoionization from a nearby source, typically O-B stars or by the flash emission from a supernova ${ }^{49}$. Having already excluded a supernova origin for the super-remnant, we must explore whether the 'lighthouse-effect' pulse of ionising radiation from the recurrent eruptions of 12a could have grown a fossil region as large as the super-remnant.

Perhaps the simplest form of a photoionization region is that of a static 'Strömgren' sphere. For this analysis, we will assume that the density and temperature limits for the

ISM around the nova $\left(\mathrm{n}<50 \mathrm{~cm}^{-3}\right.$ and $\left.\mathrm{T}<9,000 \mathrm{~K}\right)$, determined for the outer-shell, apply but we will also impose a density limit of $\mathrm{n}>1 \mathrm{~cm}^{-3}$. We note that the equivalent mid-disk region of the Milky Way has an electron density of $\sim 20 \mathrm{~cm}^{-3(50)}$. In addition, we assume that the nova emits a spectrum of solely $15 \mathrm{eV}$ photons for two weeks each year (the 
timescale of each eruption; and an overestimate of the number of ionising photons produced) and that Case B recombination applies. Under these restrictions, to grow a Strömgren sphere to a radius of $67 \mathrm{pc}$ would require a nova bolometric luminosity $>1.3 \times 10^{6}$ Lsun (assuming that all radiation blueward of the Lyman limit was tasked solely with ionizing the ISM) - at least an order of magnitude higher than actually observed ${ }^{13}$. If such a fossil were still to be growing then the energy output required for each nova eruption must be even larger.

Another possibility could be a photoionization 'echo' from each of the nova flashes. Such a phenomenon is similar to a light echo, but is delayed by the recombination timescales of the surrounding material. A photoionization echo propagating through an inclined plane of material has been proposed to describe an apparent ring surrounding the site of SN $1987 \mathrm{~A}^{51,52}$. For reasonable assumptions of the local ISM density $\left(50 \mathrm{~cm}^{-3}\right)$, a lower limit on the recombination timescale is $\sim 2000$ years. Any given echo (light or photoionization) would typically produce a superluminally expanding ring, that fades with time as the observed radius increases. However, once we have multiple flashes, and in the photoionization case flashes that occur more frequently than the recombination timescale, the picture is different. The echo emission must always be dominated by more recent flashes. Continual flashes will instead present a limb-darkened disk of emission, not a ring.

\section{Dynamics of the remnant}

We can utilise the ground-based imaging of the super-remnant to also place constraints on the growth rate of the super-remnant. Between the BOK and LT Ho imaging there is a baseline of $8.5 \pm 0.6 \mathrm{yr}$. Over this baseline there is no significant change in the nebula, we 
can constrain the growth of the outer edge of the remnant to $<6.4 \mathrm{mas} / \mathrm{yr}(<0.02 \mathrm{pc} / \mathrm{yr}$; $<0.08 \mathrm{c} ; 23 \times 10^{3} \mathrm{~km} / \mathrm{s}$ ) [upper limit of shift $54.6 \mathrm{mas}, 1 / 5$ pixel]. If we also utilise $\mathrm{H \alpha}$ imaging from the Local Group Galaxies Survey $\left(\mathrm{LGGS}^{53}\right)$ that we can extend this baseline to $14.1 \pm 0.4 \mathrm{yr}$, which yields $\left(<0.01 \mathrm{pc} / \mathrm{yr} ;<0.05 \mathrm{c} ; 14 \mathrm{x} 10^{3} \mathrm{~km} / \mathrm{s}\right)$. Therefore, assuming no deceleration of an expanding front, these very loose velocity limits show that the remnant must have existed for at least a millennium.

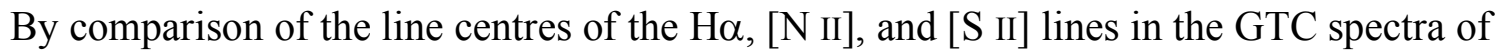
the outer-shell (to the west) and the bright knot (to the east) we find no differential radial velocity down to a five-sigma upper limit of $36 \mathrm{~km} / \mathrm{s}$. But we note that, depending upon the geometry of the super-remnant, the bulk motion at these positions might be expected to be constrained to the plane of the sky. The simulations indicate that the outer edge of the super-remnant has a power-law deceleration (Extended Data Fig. 5b), $\mathrm{v}^{\mathrm{t}} \mathrm{t}^{-0.4}$; the predicted present expansion velocity of the super-remnant is $5 \mathrm{~km} / \mathrm{s}$.

\section{Super-remnant X-ray luminosity}

To compute the present-day X-ray luminosity of the super-remnant we again employed the hydrodynamic simulations to compute a time-series of synthetic X-ray spectra, following the procedure given in (refs 54 and 55). The cells of the simulation were grouped into 30 logarithmically distributed temperature bins between $10^{4}$ and $10^{9} \mathrm{~K}$ (see Extended Data Fig. 7, top). The contribution from each bin was determined by its emission measure. The resulting 30 temperature component plasma was used as an input to XSPEC (v12.10.0c) which computed the X-ray spectrum using the MEKAL model. We assumed that the material had Solar composition and the spectrum included effects of emission from $\mathrm{C}, \mathrm{N}, \mathrm{O}, \mathrm{Ne}, \mathrm{Na}, \mathrm{Mg}, \mathrm{Al}, \mathrm{Si}, \mathrm{S}, \mathrm{Ar}, \mathrm{Ca}, \mathrm{Fe}$, and $\mathrm{Ni}$, and free-free, free- 
bound, and two-electron emission processes. Synthetic X-ray spectra, covering 0.3-10keV, were produced for all time-steps in the 100,000-eruption model (see Extended Data Fig. 7, bottom), and the evolution of the predicted X-ray luminosity is shown in Extended Data Fig. 5c.

This emission modelling shows that at all times, the majority of the super-remnant emission arises from the super-remnant outer-shell - the piled-up ISM. Other regions of the structure are far too low density (and the ejecta pile-up region too hot) to contribute significantly to the emission. Again, this supports the finding of negligible radiative cooling from the multiple ejecta, and that radiation losses do not affect the dynamics of the super-remnant.

The evolution of the emission measure (Extended Data Fig. 7, top) indicates that the luminosity of the super-remnant grows with time. However, the X-ray luminosity peaks at $\sim 1,000$ eruptions as the peak of the emission measure distribution continually shifts to longer wavelengths with time. As is illustrated in Extended Data Fig. 5c, the initial peak emission occurs in the hard X-ray (1-10keV), before shifting to softer X-rays. The post $\sim 1,000$ eruption $\mathrm{X}$-ray decline is simply due to the peak emission moving into, first, the extreme UV (10-124eV; 10,000 eruptions), then the UV (100,000 eruptions). By simple logarithmic extrapolation, the expected current emission peak will be in the infrared

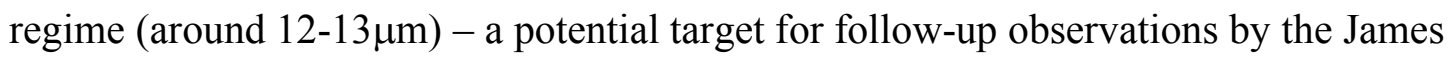
Webb Space Telescope.

The X-ray luminosity of the super-remnant peaks after approximately 1,000 eruptions at $\sim 6 \times 10^{31} \mathrm{erg} \mathrm{s}^{-1}$, before fading to $9 \times 10^{29} \mathrm{erg} \mathrm{s}^{-1}$ after 100,000 eruptions. Using a powerlaw extrapolation, the expected present-day X-ray luminosity is $3 \times 10^{29} \mathrm{erg} \mathrm{s}^{-1}$. At all 
times (see below), the X-ray luminosity of the super-remnant lies well below current detection capabilities.

\section{Archival X-ray observations}

To constrain the X-ray emission of the proposed nova super-remnant, we examined the available archival X-ray data for the two most sensitive (and relevant) telescopes $X M M$ Newton and Chandra. In case of Chandra, there are only three archival observations that contain the nova position, but at the very edge of their fields of view: ObsId 17012 (50ks nominal exposure), 17013 (45ks) and 17637 (10ks). In all three, the super-remnant is located (a) partly off the edge of the detector and (b) so far off-axis that we are not able to benefit from the superior (on-axis) spatial resolution of Chandra. Therefore, those data are significantly less useful for our objective than the XMM-Newton observations and we do not consider them in the following analysis.

In case of XMM-Newton, there exist four archival observations with a total of $211 \mathrm{ks}$ effective exposure; i.e. after considering dead-time and exposure mask vignetting. Due to the superior collecting area of $X M M-N e w t o n$ this exposure results in significantly more photons than Chandra would have collected in the same time. The observations were taken in the following years (with ObsIDs in parentheses): 2002 (0109270301), 2007 (0402561501), 2015 (0763120301), and 2016 (0763120401). The 2002 and 2007 observations were employed to produce one of the X-ray catalogues used to rule out a SNR origin for the super-remnant ${ }^{25}$. The inclusion of the 2015 and 2016 X-ray data results in more than a tripling of the effective exposure time. After conducting substantial testing, we decided to use an elliptical source count extraction region, rather than an annulus, to study the super-remnant emission. The main 
reason for this is the relatively large $X M M-N e w t o n$ PSF, which, at the relatively small size of the super-remnant, would not give us an annulus shape for a detectable source. Fortunately, there are no known X-ray (point) sources near 12a in quiescence. Note that with an estimated quiescent X-ray luminosity of $10^{2.5} \operatorname{Lsun}^{(56)}, 12 \mathrm{a}$ is yet to be detected in X-rays outside eruptions. We only use the EPIC pn detector due to its higher response at lower energies.

Combining all XMM-Newton observations we detect no significant X-ray emission from the nova super-remnant. We derive a count rate upper limit of $6.74 \times 10^{-4} \mathrm{ct} / \mathrm{s}$ on the $3 \sigma$ confidence level, using a Bayesian approach ${ }^{57}$. Assuming a simple $1 \mathrm{keV}$ plasma with Solar abundance and foreground absorption, as expected from a young SNR, this rate corresponds to an unabsorbed luminosity of $<9 \times 10^{34} \mathrm{erg} \mathrm{s}^{-1}$ at the M31 distance of 770kpc. This limit is five orders of magnitude greater than the expected present-day Xray luminosity of the super-remnant.

\section{Methods references}

31. Darnley, M. J., Williams, S. C., Bode, M. F., Henze, M., Ness, J.-U., Shafter, A. W., Hornoch, K., Votruba, V. A remarkable recurrent nova in M31: The optical observations. Astron. Astrophys. 563, L9 (2014).

32. Henze, M., Ness, J.-U., Darnley, M. J., Bode, M. F., Williams, S. C., Shafter, A. W., Kato, M., Hachisu, I. A remarkable recurrent nova in M31: The X-ray observations. Astron. Astrophys. 563, L8 (2014).

33. Henze, M., Darnley, M. J., Kabashima, F., Nishiyama, K., Itagaki, K., Gao, X. A remarkable recurrent nova in M31: The 2010 eruption recovered and evidence of a sixmonth period. Astron. Astrophys. 582, L8 (2015). 
34. Shafter, A. W., Henze, M., Rector, T. A., Schweizer, F., Hornoch, K., et al. Recurrent Novae in M31. Astrophys J. Suppl. Ser. 216, 34 (2015).

35. Steele, I. A., Smith, R. J., Rees, P. C., Barker, I. P., Bates, S. D. The Liverpool Telescope: performance and first results. Proc. SPIE. 5489, 679-692 (2004).

36. Tody, D. IRAF in the Nineties. Astronomical Data Analysis Software and Systems II. (San Francisco, CA: ASP), 173-183 (1993).

37. Stetson, P. B. DAOPHOT - A computer program for crowded-field stellar photometry. Publ. Astron. Soc. Pacif. 99, 191-222 (1987).

38. Dressel, L. Wide Field Camera 3 Instrument Handbook, Version 9.0. Baltimore: STScI (2017).

39. Currie, M. J., Berry, D. S., Jenness. T., Gibb, A. G., Bell., G. S., Draper, P. W. Starlink Software in 2013. Astronomical Data Analysis Software and Systems XXIII. (San Francisco, CA: ASP), 391-394 (2014).

40. Chonis, T. S., Hill, G. J., Lee, H., Tuttle, S. E., Vattiat, B. L. LRS2: design, assembly, testing, and commissioning of the second-generation low-resolution spectrograph for the Hobby-Eberly Telescope. Proc. SPIE. 99084, 1-28 (2016).

41. Davis, B. D., Ciardullo, R., Jacoby, G. H., Feldmeier, J. J.; Indahl, B. L. The True Luminosities of Planetary Nebulae in M31's Bulge: Massive Central Stars from an Old Stellar Population. Astrophys J. 863, 189 (2018).

42. Hamuy, M., Walker, A. R., Suntzeff, N. B., Gigoux, P., Heathcote, S. R., Phillips, M. M. Southern spectrophotometric standards. Publ. Astron. Soc. Pacif. 104, 533-552 (1992). 
43. Slavin, A. J., O’Brien, T. J., Dunlop, J. S. A deep optical imaging study of the nebular remnants of classical novae. Mon. Not. R. Astron. Soc. 276, 353-371 (1995).

44. Freedman, W. L., Madore, B. F., An empirical test for the metallicity sensitivity of the Cepheid period-luminosity relation. Astrophys J. 365, 186-194 (1990).

45. Vaytet, N. M. H., O’Brien, T. J., Bode, M. F. Swift Observations of the 2016 Outburst of the Recurrent Nova RS Ophiuchi. II. One-dimensional Hydrodynamical Models of Wind-driven Shocks. Astrophys J. 665, 654-662 (2007).

46. Lloyd, H. M., O’Brien, T. J., Bode, M. F. Shaping of nova remnants by binary motion. Mon. Not. R. Astron. Soc. 284, 137-147 (1997).

47. Wareing, C. J., O’Brien, T. J., Zijlstra, A., Kiwtter, K. B., Irwin, J., et al. The shaping of planetary nebula Sh 2 - through interaction with the interstellar medium. Mon. Not. R. Astron. Soc. 366, 387-396 (2006).

48. Bode, M. F., Kahn, F. D. A model for the outburst of nova RS Ophiuchi in 1985. Mon. Not. R. Astron. Soc. 217, 205-215 (1985).

49. Brandt, J. C., Stecher, T. P., Crawford, D. L., Maran, S. P. The Gum Nebula: Fossil Strömgren Sphere of the Vela X Supernova. Astrophys J. 163, L99-L104 (1971).

50. Merrifield, M. R. The rotation curve of the Milky Way to $2.5 \mathrm{R}_{0}$ from the thickness of the H I layer. Astron. J. 103, 1552-1563 (1992).

51. Jakobsen, P., Albrecht, R., Barbieri, C., Blades, J. C., Boksenberg, A., et al. First results from the faint object caera: SN 1987A. Astrophys J. 369, L63-L66 (1991). 52. Panagia, N., Gilmozzi, R., Macchetto, F., Adorf, H.-M., Kirshner, R. P. Properties of the SN 1987A circumstellar ring and the distance to the Large Magellanic Cloud. Astrophys J. 380, L23-L26 (1991). 
53. Massey, P., McNeill, R. T., Olsen, K. A. G., Hodge, P. W., Blaha, C., et al. A Survey of Local Group Galaxies Currently Forming Stars. III. A Search for Luminous Blue Variables and Other Ha Emission-Line Stars. Astron. J. 134, 2474-2503 (2007).

54. Vaytet, N. M. H. Numerical Modelling of the 2006 Outburst of RS Ophiuchi. PhD Thesis, University of Manchester. (2009)

55. Vaytet, N. M. H., O’Brien, T. J., Page, K. L., Bode, M. F., Lloyd, M., Beardmore, A. P. Swift Observations of the 2006 Outburst of the Recurrent Nova RS Ophiuchi. III. X-ray Spectral Modeling. Astrophys J. 740, 5 (2011).

56. Kato, M., Saio, H., Hachisu, I. A Millennium-long Evolution of the 1 yr Recurrent Period Nova- Search for any Indication of the Forthcoming He Flash. Astrophys J. 844, 143 (2018).

57. Kraft, R. P., Burrows, D. N., Nousek, J, A. Determination of Confidence Limits for Experiments with Low Numbers of Counts. Astrophys J. 374, 334-355 (1991).

\section{Data Availability}

All relevant data generated or analysed during this study are available from the corresponding author on reasonable request.

\section{Extended Data Figure Legends}

\section{Extended Data Figure 1. Additional mutli-wavelength imaging of the super-remnant}

region. (a), the Steward $2.3 \mathrm{~m}$ Bok Telescope $\mathrm{H \alpha}$ image that allowed the association between the nebulosity and M31N 2008-12a to be made. Image orientation is as Fig. 1 but the image is $80^{\prime \prime} \times 80^{\prime \prime}$. (b-d), Hubble Space Telescope Wide Field Camera 3 broad- 
band filter images of the region around M31N 2008-12a. Image sizes are $40^{\prime \prime} \times 40^{\prime \prime}$. These three panels show the (b) F275W (ultraviolet), (c) F475W (optical), and (d) F814W (optical) filters. The white contours in the (b) show iso-flux regions as derived from the ground-based $\mathrm{H} \alpha+[\mathrm{N} \mathrm{II}]$ image. As (b-d) were taken toward the end of the 2015 eruption, the nova can be seen in the images. The F275W image clearly illustrates the lack of bright UV sources within the super-remnant.

\section{Extended Data Figure 2. Hobby Eberly Telescope flux calibrated spectrum of the} super-remnant outer-shell. As with the GTC spectrum of the same region (Fig. 2), there is negligible continuum and hydrogen Balmer emission lines and nebular lines of [N II], [O II], and [S II]. The mean spectral resolution of the "UV" and "orange" arms are 1.68

and $4.04 \AA$, respectively. Gaps in the spectrum indicate areas where significant skyline subtraction residuals remained.

\section{Extended Data Figure 3. Comparison of the results from the hydrodynamic} modelling using a range of spatial resolutions. The blue and green lines indicate simulations of 20 eruptions with spatial resolutions of 0.02 and $0.2 \mathrm{AU}$, respectively, while the red and black lines indicate simulations of 100 eruptions with resolution 0.2 and 0.4 AU, respectively. (a) Gas density radial distribution, the lower black dotted horizontal line indicates the ISM density, with the upper dotted line showing the consistent peak density of the super-remnant shell. (b) Gas pressure radial distribution. (c) Gas velocity radial distribution. (d) Gas temperature radial distribution.

\section{Extended Data Figure 4. The effect of radiative cooling on the super-remnant} dynamics. Panels as Extended Data Fig. 3. The close comparison between the results of simulations of 1,000 eruptions without radiative cooling (black) and with radiative 
cooling (blue).

Extended Data Figure 5. Additional results of the hydrodynamic simulations of the interacting ejecta of multiple recurrent nova eruptions. (a), the mass growth of the super-remnant outer-shell for up to 100,000 eruptions (see key). The diagonal dotted line illustrates a power-law extrapolation of the outer-shell mass to further eruptions. The upper/lower solid grey lines indicate the growth of the outer-shell mass for higher/lower ISM densities, respectively. The horizontal line marks the predicted outer-shell mass at the current extent of the super-remnant. (b), the evolution of the expansion velocity of the outer shell (black) compared to the mean velocity within the ejecta pile-up region (red). The diagonal dotted lines indicate power-law extrapolations, the horizontal line the initial injection velocity, the vertical line the predicted current epoch. (c), the evolution of the X-ray (0.3-10keV) luminosity of the super-remnant (black), the hard (1-10keV; red) and soft (0.3-1keV; blue) components are shown for information along with the hardness ratio (hard/soft; right-hand axis) evolution; for each, the dotted line indicates a power-law extrapolation to later times. The horizontal dotted black line indicates the $3 \sigma$ upper limit from the XMM-Newton observations.

Extended Data Figure 6. The full (uncooled) simulations of 100,000 eruptions. Panels as Extended Data Fig. 4.

Extended Data Figure 7. Super-remnant X-ray emission modelling. In both panels the cyan, blue, green, red, and black lines indicate simulations of 10, 100, 1,000, 10,000, and 100,000 eruptions, respectively. Top: Contribution to the super-remnant emission as a function of temperature (in units of kT). The vertical dotted line indicates the lower- 
limit $(0.08 \mathrm{keV})$ cut-off for input into XSPEC. Bottom: The resultant synthetic X-ray spectra of the super-remnant $(0.3-10 \mathrm{keV})$. 

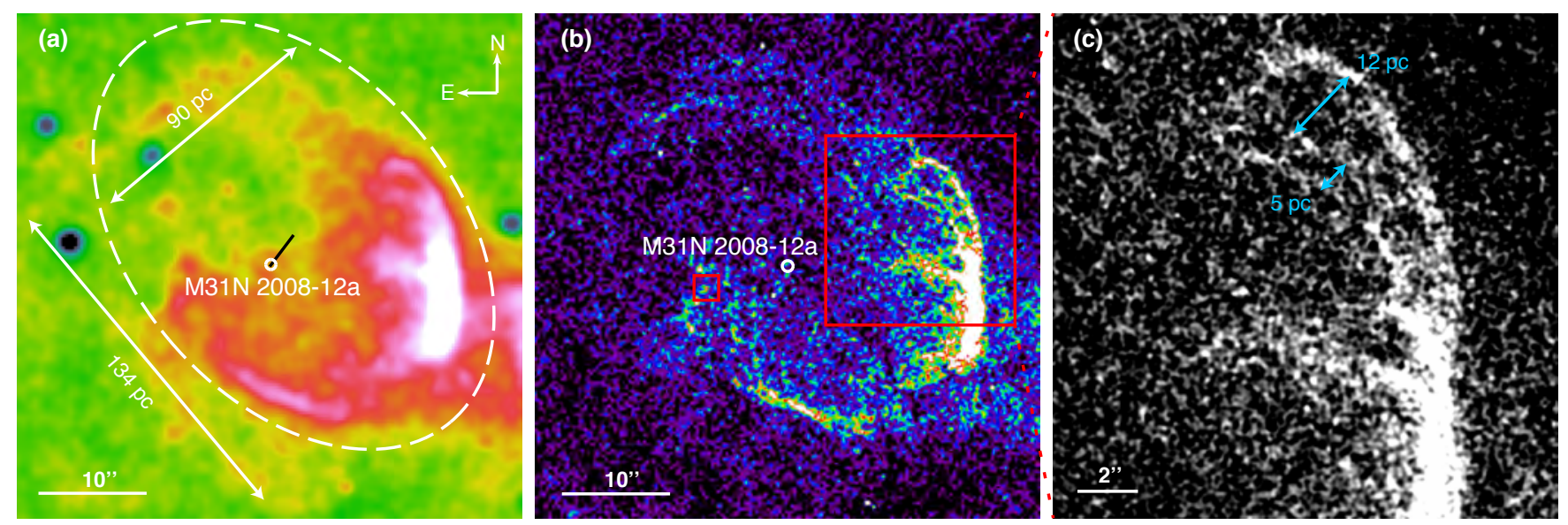


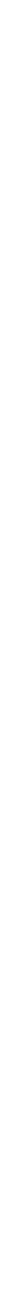



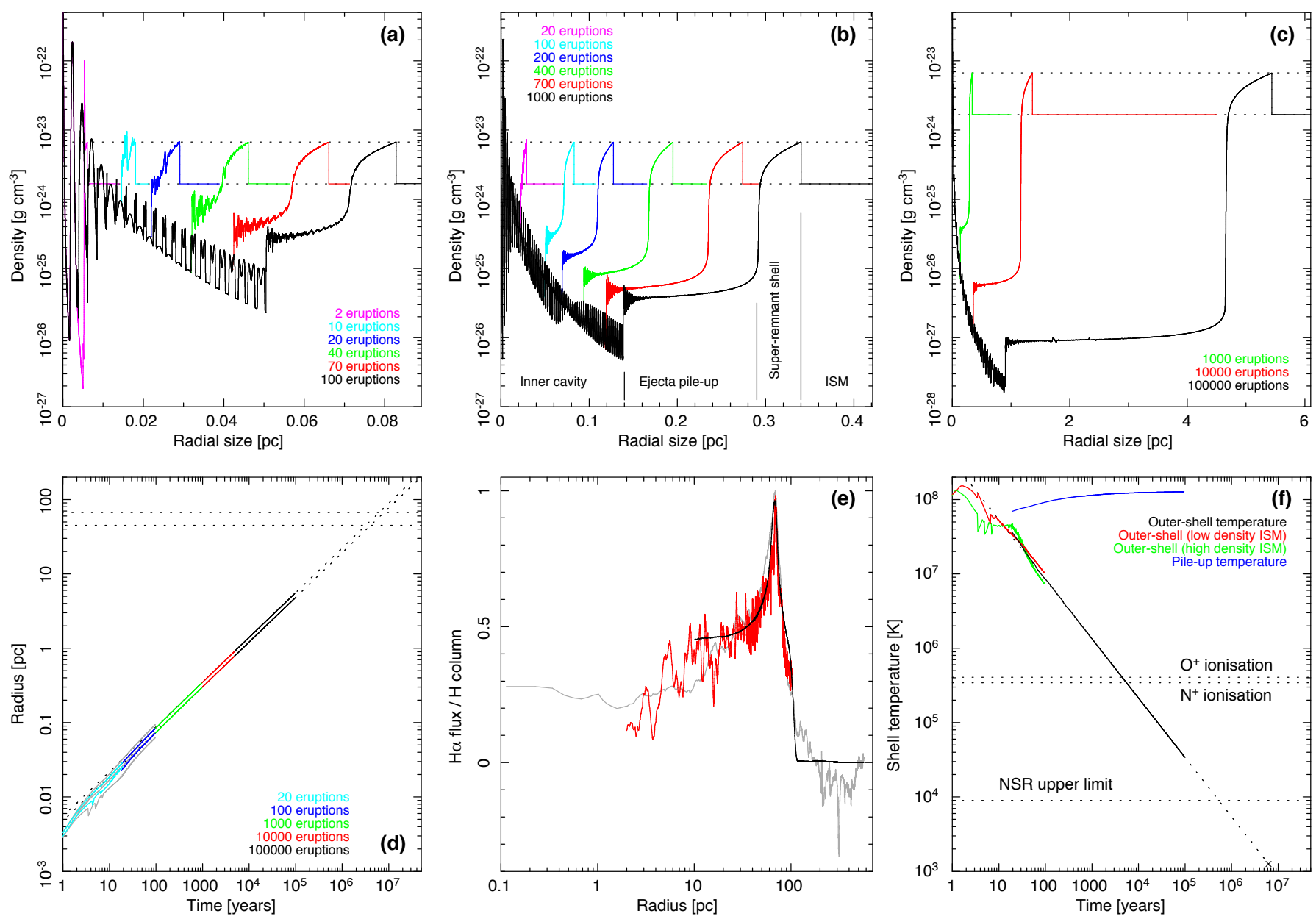

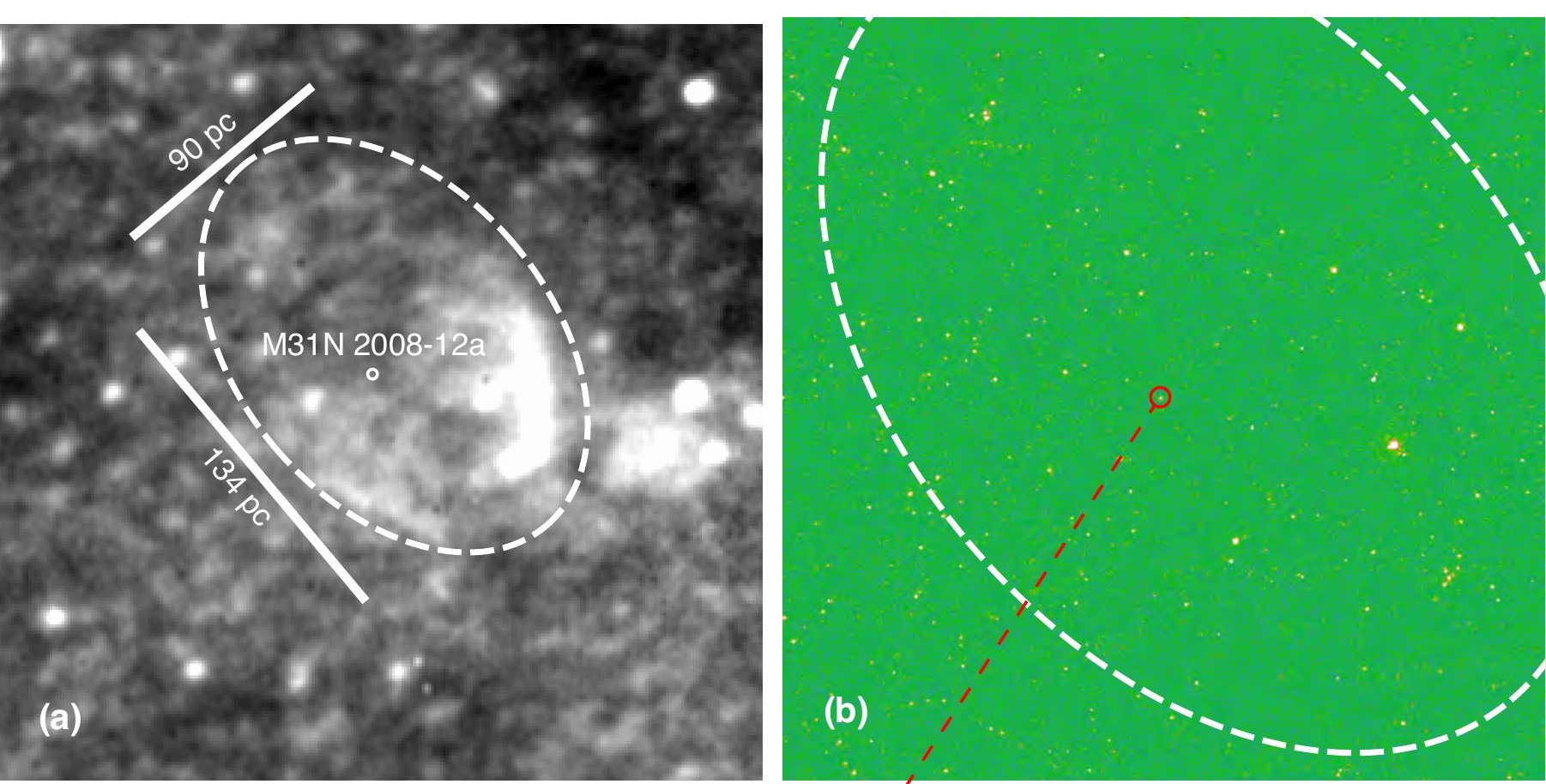

M31N 2008-12a'

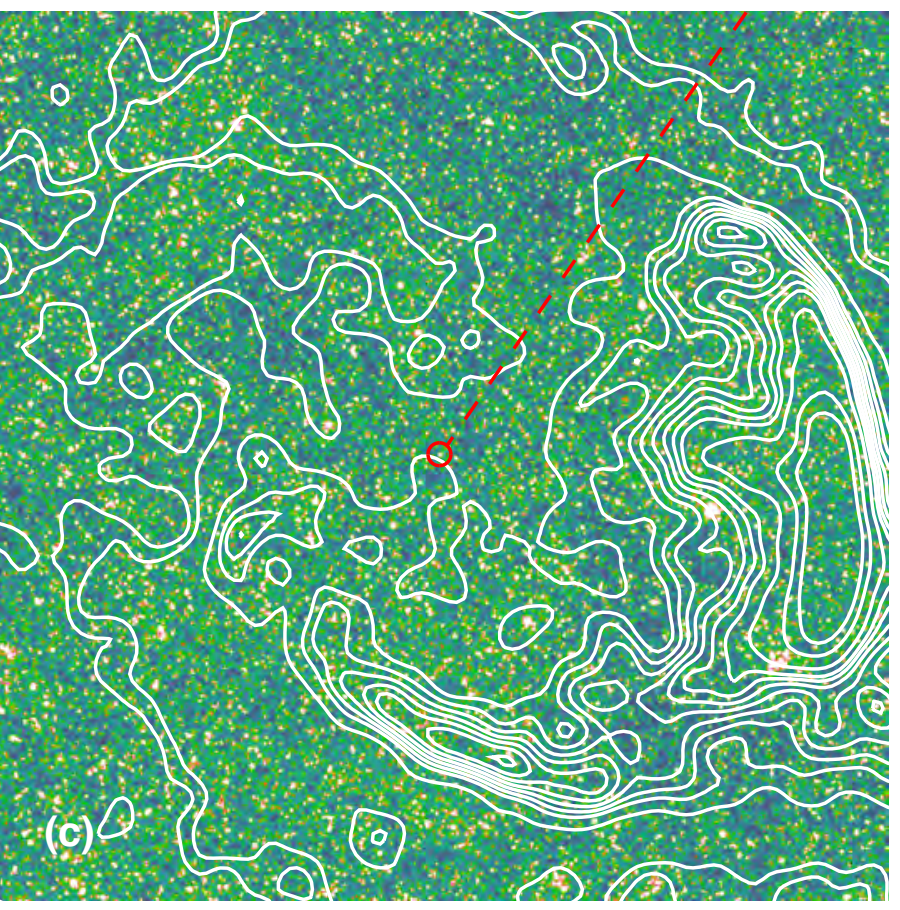

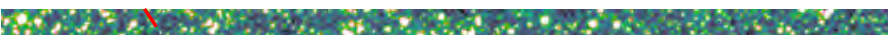

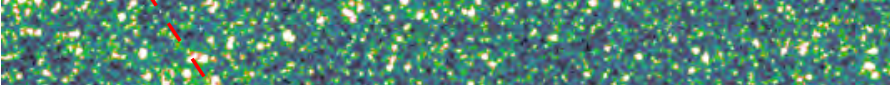

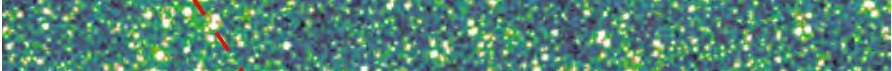

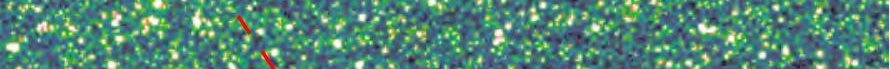

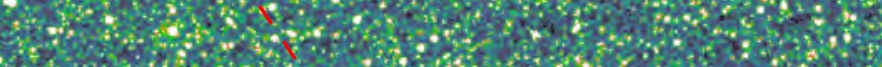

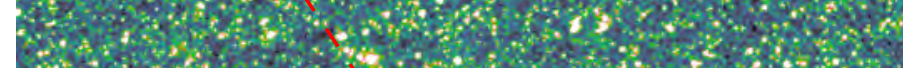

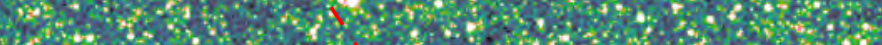

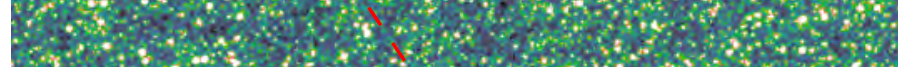

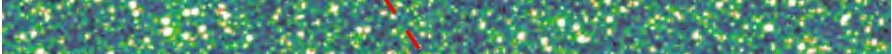

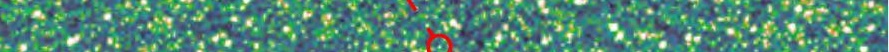

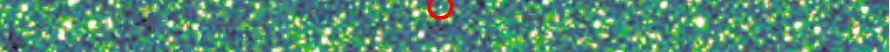

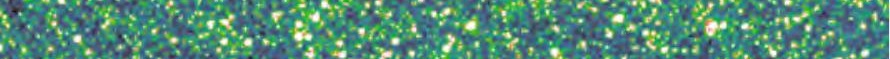

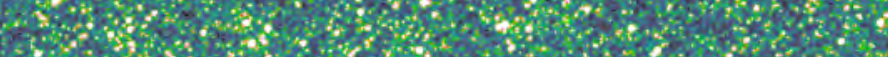

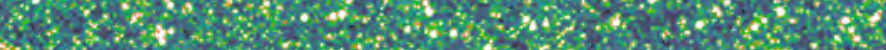

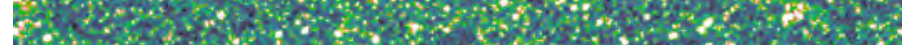

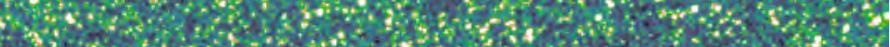

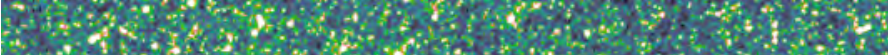

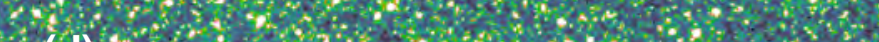

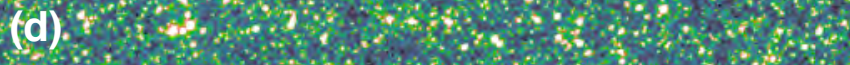

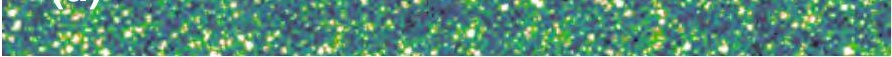




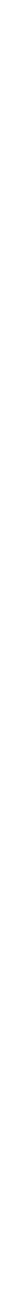



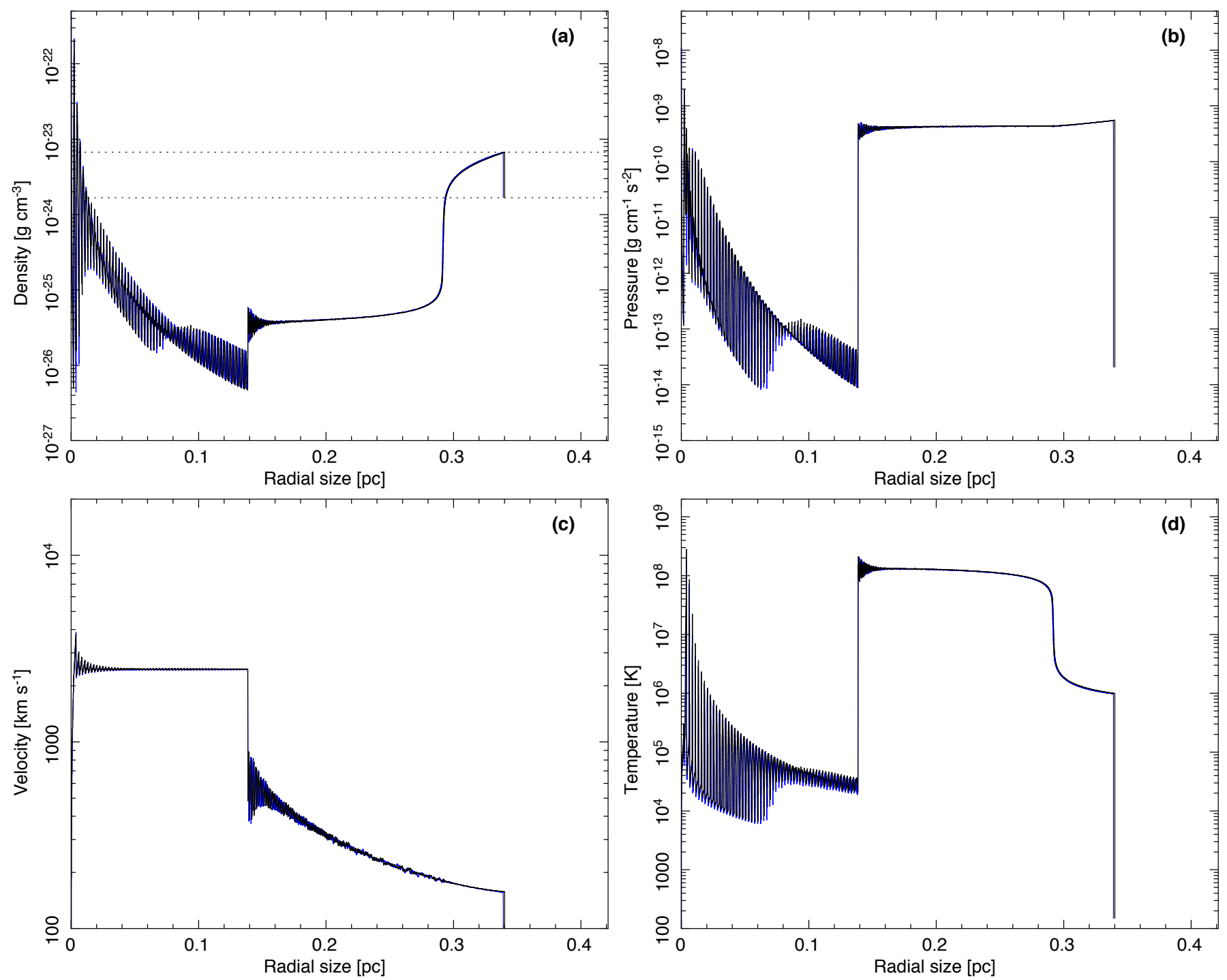

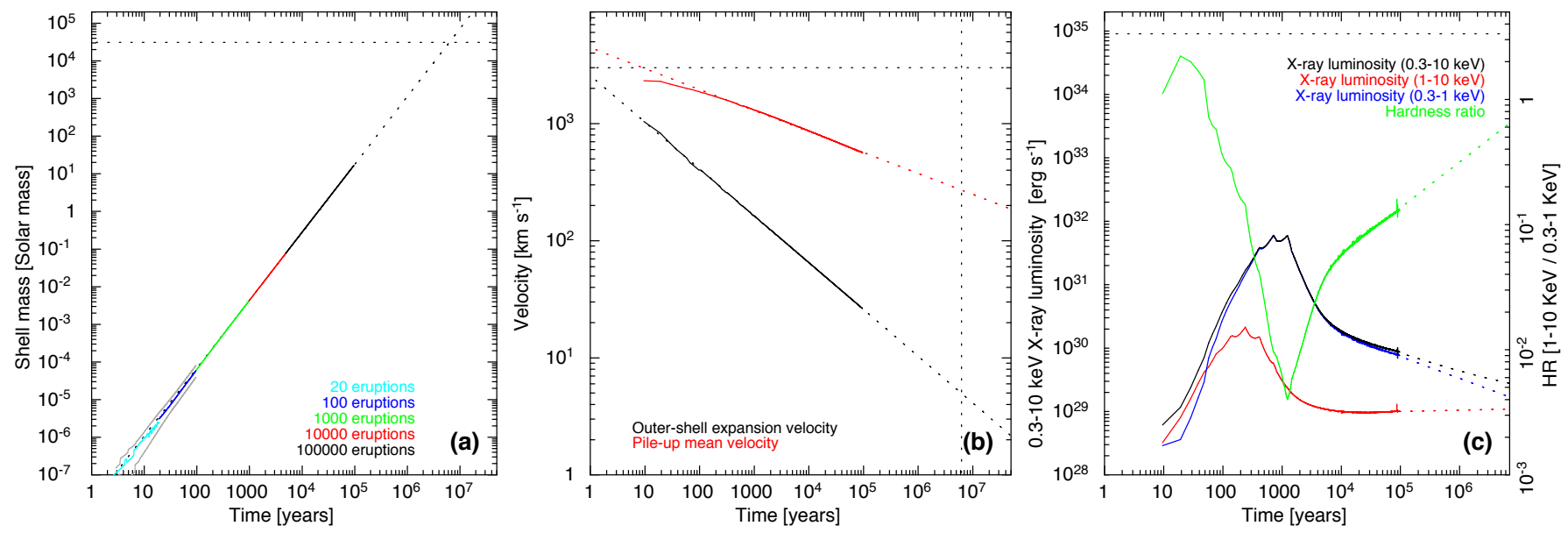

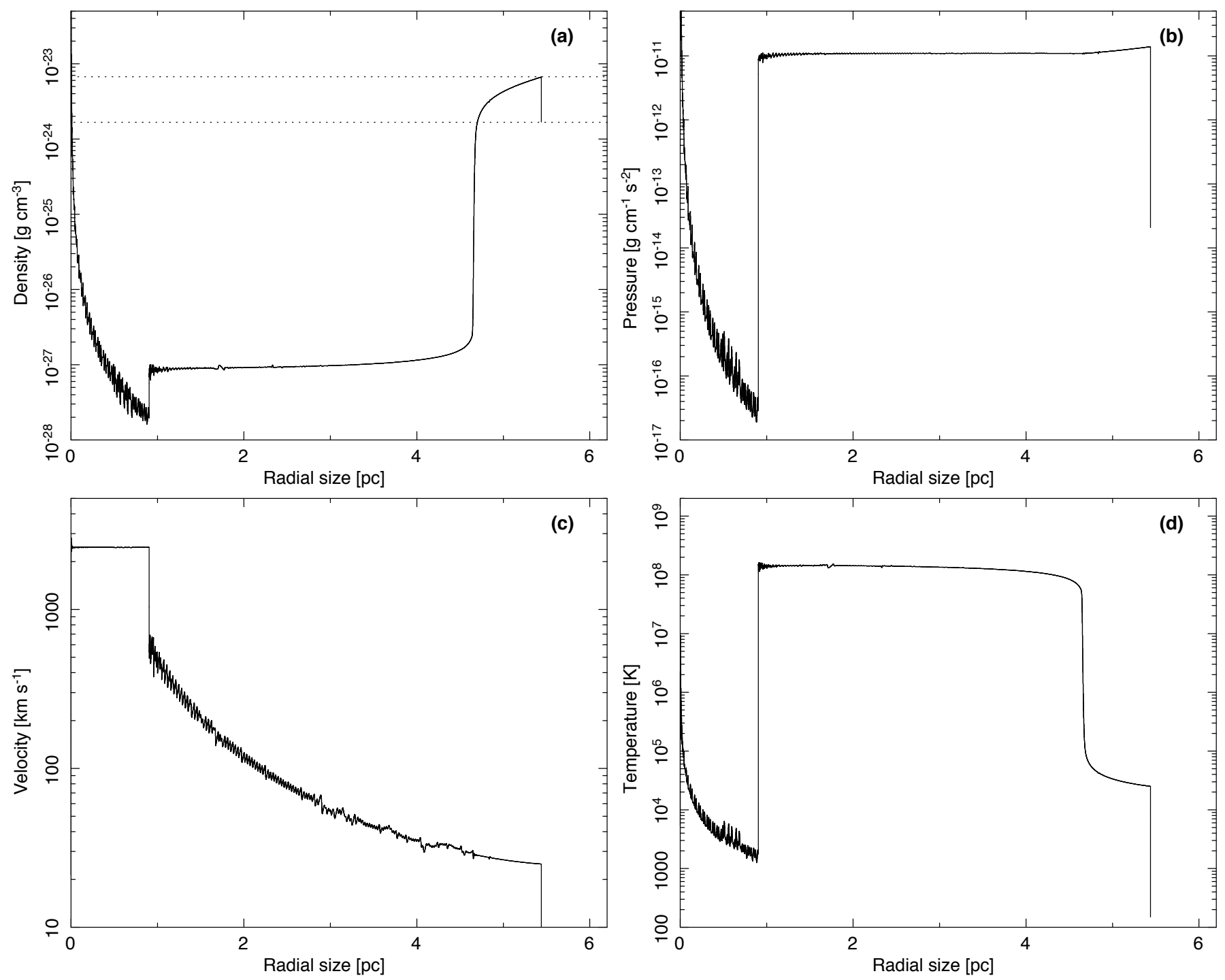


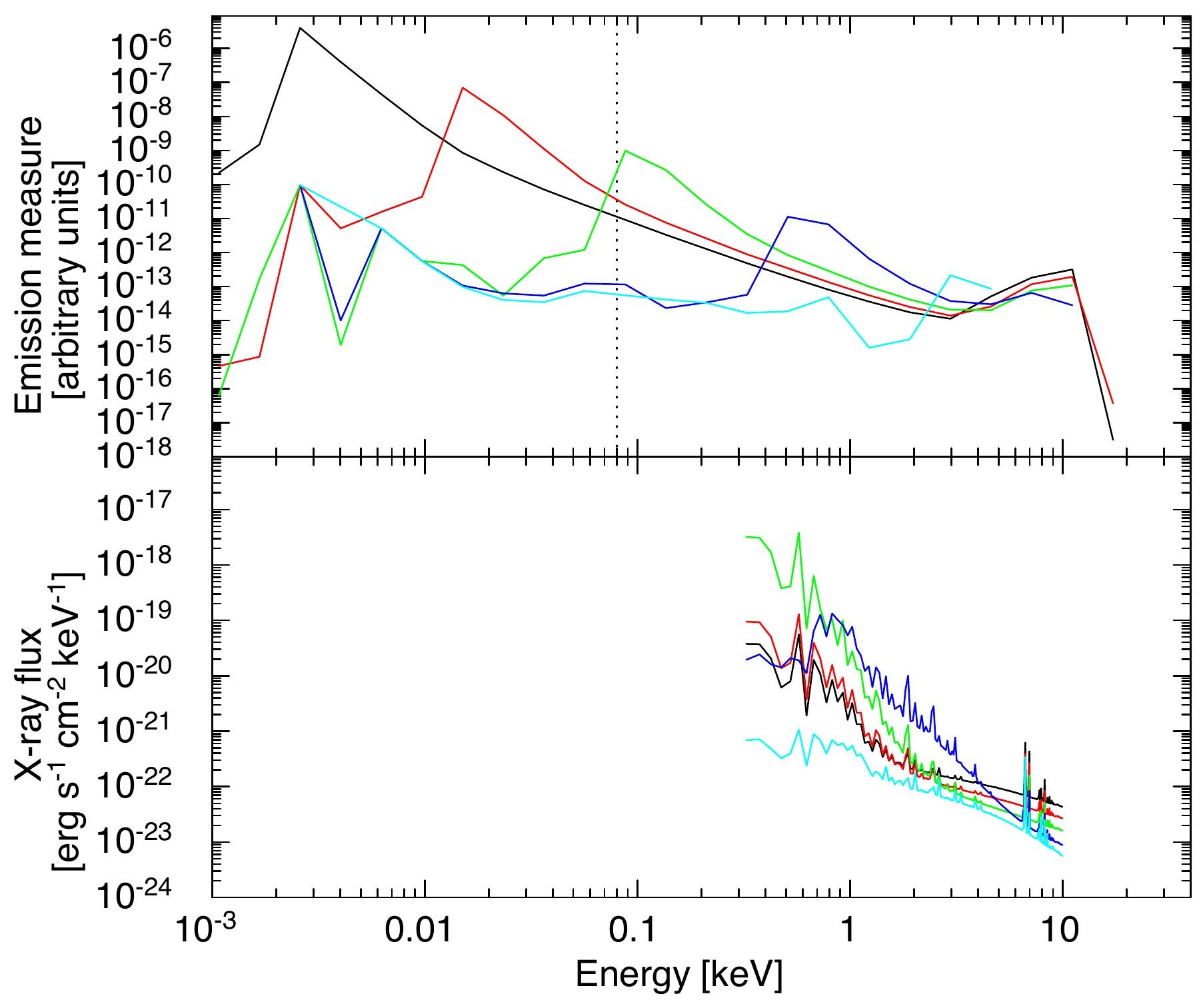

